\title{
Transmission of Listeria monocytogenes from raw chicken meat to cooked chicken meat through cutting boards
}

\begin{abstract}
Listeria monocytogenes (L. monocytogenes) is a food-borne pathogen contaminating poultry products. Ready-to-eat (RTE) cooked chicken meat can easily be contaminated with L. monocytogenes in post-processing activities. This study aimed to determine transmission of L. monocytogenes from raw chicken meat to hot and cooled chicken meat through polyethylene and wooden cutting boards. Raw chicken breast samples were purchased from retail markets and were artificially contaminated with $\mathrm{L}$. monocytogenes at concentration of $7.35 \pm 0.22 \log \mathrm{CFU} / \mathrm{ml}$. Contaminated raw samples were placed on polyethylene and wooden cutting boards to simulate bacterial transfer to cutting boards. Cooked chicken samples (hot and cooled) were then placed on the same cutting boards to simulate transfer of bacteria from cutting boards to cooked meat. L. monocytogenes successfully attached to polyethylene and wooden cutting boards and recovered after holding time up to $1 \mathrm{~h}$. Transmissions of L. monocytogenes to cooled cooked samples from both types of cutting boards were relatively higher than hot cooked samples. Moreover, transfer rates of L. monocytogenes from wooden cutting boards at holding time of $1 \mathrm{~h}$ to both cooled and hot cooked samples were lower than those from polyethylene cutting board. It is recommended to use different cutting boards for raw and cooked materials and apply detergents and hot water for cleaning procedure to eliminate L. monocytogenes attached to the cutting boards and prevent cross-contamination of final products.
\end{abstract}

Keyword: Cutting boards; Cooked chicken meat; Listeria monocytogenes; Transmission 